\title{
Edge Strength Functions as Shape Priors in Image Segmentation
}

\author{
Erkut Erdem, Aykut Erdem, and Sibel Tari \\ Middle East Technical University, Department of Computer Engineering, Ankara, \\ TR-06531, TURKEY, \\ \{erkut, aykut\}@ceng.metu.edu.tr, stari@metu.edu.tr
}

\begin{abstract}
Many applications of computer vision requires segmenting out of an object of interest from a given image. Motivated by unlevel-sets formulation of Raviv, Kiryati and Sochen [8] and statistical formulation of Leventon, Grimson and Faugeras [6], we present a new image segmentation method which accounts for prior shape information. Our method depends on Ambrosio-Tortorelli approximation of Mumford-Shah functional. The prior shape is represented by a by-product of this functional, a smooth edge indicator function, known as the "edge strength function", which provides a distance-like surface for the shape boundary. Our method can handle arbitrary deformations due to shape variability as well as plane Euclidean transformations. The method is also robust with respect to noise and missing parts. Furthermore, this formulation does not require simple closed curves as in a typical level set formulation.
\end{abstract}

\section{Introduction}

In many vision applications, one searches an object of interest whose pose may vary and whose shape may exhibit variability. Deliniating the object boundary correctly and estimating the pose becomes particularly challenging when corrupting influences due to missing regions and noise appear. As a remedy, use of prior shape models are considered. During the last five years, quite interesting works addressing shape prior integration directly into segmentation process appeared $[4-6,8,9,11]$.

These methods differ in terms of

- How they represent shape prior;

- Boundary detection rule which forms the backbone;

- Extension of the boundary detection rule to allow the influence of the prior;

- Computation.

In Cremers et.al. [5], a variational framework is used for the integration of shape statistics and segmentation. Shape boundaries are represented explicitly as spline curves. Aligned shape variability is captured by a Gaussian distribution model whose mean and covariance matrix are computed from a group of splines. A shape energy that maximizes the shape probability is combined with MumfordShah [7] segmentation energy and minimized by applying gradient descent. 
In Leventon et.al. [6], a shape boundary is embedded as the zero level curve of a level set function (distance function). Shape prior is represented via coefficients of the principal components computed from a group of distance functions whose zero-levels correspond to the various appearances of a shape of interest. A two step procedure is employed. First, the level set function is evolved such that its zero level curve converges to the shape boundary. Second, pose and shape variables are computed via MAP estimation on the Gaussian probability space.

In Tsai et.al. [11], a shape based curve evolution technique is proposed. The implicit shape representation proposed by Leventon et.al. [6] is embedded into region-based active contour models as in [3]. Again a two step procedure is employed. However, pose and shape variables are computed by applying gradient descent.

In Chen et. al. [4], prior shape is represented by the average of aligned contours. A shape term which measures the similarity between evolving and prior contours is added into a variational active contours framework. Hence, evolution of the active contour is controlled by a force which depends on both image gradients and prior shape.

In Rousson et.al. [9], a probabilistic approach to generate shape priors using level set representations which can also handle local shape variability is proposed. From a set of training samples, represented as level sets, a probability density function is constructed by maximum likelihood estimation. A shape energy is defined and incorporated into a level set based segmentation method depending on the proposed model.

In Raviv et.al. [8], shape variability is ignored. Quite elegant formulation is obtained by utilizing a generalized cone whose cross sections are the various appearances of a given object under pose changes. This cone also functions as a level set function which evolves according to a constraint derived from the prior. The cost function is an extension of Chan-Vese approximation [3] of MumfordShah functional with a shape prior term.

Inspired by Raviv et.al. [8] and Leventon et.al. [6], we present a new method for shape prior incorporation into segmentation process. Backbone of our method is Ambrosio-Tortorelli [1] approximation (AT) of Mumford-Shah functional. We employ a by-product of this functional, a smooth edge indicator function which is known as the "edge strength function" as a distance-like surface which embeds the shape boundary. Shape similarity term which is a normalized difference between "deformed" shape prior and the evolving edge strength function is added to Ambrosio-Tortorelli functional.

The edge strength function has some nice properties which makes it a quite versatile tool for different vision tasks. Despite its shortcomings as a segmentation tool, it has been proven to be quite useful in capturing essential shape characteristics [10] and recently applied to object recognition very successfully [2]. An interesting property of the edge strength function is that it encodes the local symmetry information [10]. This makes possible the integration of boundary and local symmetry information and design shape energies which will force morphological equivalence. As an example, it is possible to change the shape en- 
ergy by simply adding a weight that is proportional to local symmetry strength. Furthermore, the embedding provided by the edge strength function does not require simple closed curves as in a typical level set formulation.

The paper is organized as follows. Section 2 is on Ambrosio-Tortorelli functional. Representation of a set of prior shapes is explained in Section 3. In Section 4, the combined energy and its minimization is discussed. Experimental results are presented in Section 5. Finally, Section 6 is the summary.

\section{Ambrosio-Tortorelli Segmentation Functional}

A prototype for energy based minimization is Mumford and Shah [7] approach. In this approach, image segmentation problem is formulated as a functional minimization via which a piecewise smooth approximation of an image and a set of discontinuity locus corresponding to object boundaries are to be recovered. The energy to be minimized is:

$$
E_{M S}=\alpha \iint_{R \backslash \Gamma}\|\nabla u\|^{2} d x d y+\beta \iint_{R}(u-g)^{2} d x d y+\operatorname{length}(\Gamma)
$$

where $R \subset \Re^{2}$ is connected, bounded, open subset representing the image domain, $g(x, y)$ is an image, $\Gamma$ is a curve segmenting $R, u(x, y)$ is the smoothed image, $\alpha$ and $\beta$ are the weights. Let $\sigma=\sqrt{\frac{\alpha}{\beta}}$. Then, $\sigma$ may be interpreted as the smoothing radius in $R \backslash \Gamma$. With $\sigma$ fixed, the higher the value of $\alpha$, the lower the penalty for length $(\Gamma)$, hence the more detailed is the segmentation. Unknown edge set $\Gamma$ makes the minimization mathematically difficult. A convenient approximation is suggested by Ambrosio and Tortorelli in [1] where they introduce a smooth edge indicator function $v(x, y)$ which is more convenient than the original edge indicator. On the edges, $v(x, y) \rightarrow 1$ and on the smooth regions $v(x, y) \rightarrow 0$. Ambrosio-Tortorelli functional

$$
E_{A T}=\iint_{R}\left(\alpha\left((1-v)^{2}\|\nabla u\|^{2}\right)+\beta(u-g)^{2}+\left(\frac{\rho}{2}\|\nabla v\|^{2}+\frac{v^{2}}{2 \rho}\right)\right) d x d y
$$

is shown to converge to the original functional as $\rho \rightarrow 0$.

\section{Representation of the Prior Shape}

For the proper choice of $\rho$, edge strength function provides a smooth embedding surface whose one-level curve correspond to shape boundary which is not necessarily a simple closed curve(Fig. 1). Prior edge strength function $v_{p}(x, y)$ can be computed from a binary prior image as the minimizer of the following energy $[10]$ 


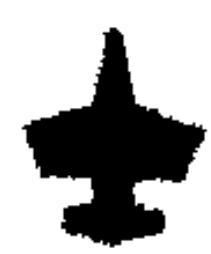

(a)

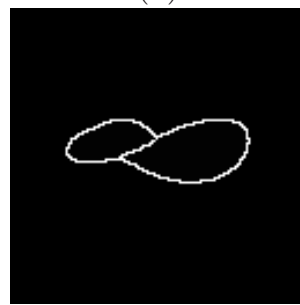

(c)

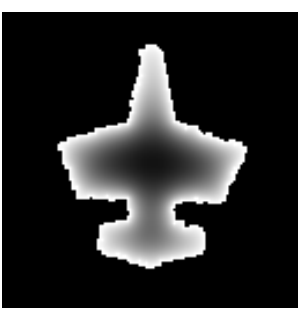

(b)

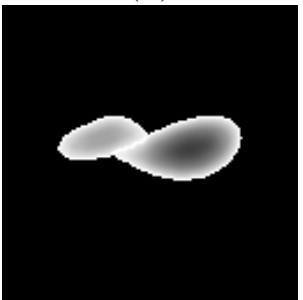

(d)

Fig. 1. (a) An airplane silhoutte. (b) Corresponding edge strength function computed with $\rho=32$. (c) a line drawing with self intersections. (d) Corresponding edge strength function computed with $\rho=16$.

$$
\frac{1}{2} \iint \rho\|\nabla v\|^{2}+\frac{v^{2}}{\rho} d x d y
$$

subject to $v=1$ on the shape boundary.

A quite curious property of the edge strength function computed with large $\rho$ is that the local symmetry information is encoded via differential properties. Consider Fig. 2 which displays the quantity $1-\left|\frac{d}{d s}\|\nabla v\|\right| \mid$ where

$$
\left|\frac{d}{d s}\|\nabla v\|\right|=\frac{\left|\left(v_{y}^{2}-v_{x}^{2}\right) v_{x y}-v_{x} v_{y}\left(v_{y y}-v_{x x}\right)\right|}{\|\nabla v\|^{2}}
$$

If a shape does not exhibit variations other than pose, $v_{p}(x, y)$ captures the prior information. Suppose we are given an ensemble of pose and scale aligned shapes $^{1}$ whose boundaries are given by the curves $\Gamma_{p_{1}}, \cdots, \Gamma_{p_{n}}$ respectively. Following an idea presented by Leventon et.al. [6] and later adopted in [11], we use coefficients of the principal components as shape variability parameters. Specifically, let $v_{p_{1}}(x, y), \cdots, v_{p_{n}}(x, y)$ be an ensemble of prior edge strength functions. The mean edge strength function $\bar{\Phi}(x, y)$ is the ordinary average of $v_{p_{1}}(x, y), \cdots, v_{p_{n}}(x, y)$. Let $\Phi_{1}, \cdots, \Phi_{n}$ be the principal components computed by Karhunen-Loeve Transformation, then a possible shape from this ensemble has

\footnotetext{
1 The alignment algorithm proposed in [11] is used in the experiments.
} 


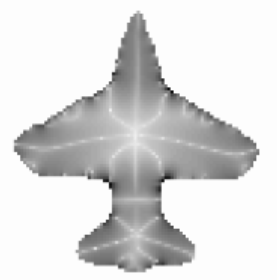

Fig. 2. Differential properties of $v$ capture local symmetry strength.

$$
\Phi_{\mathbf{w}}(x, y)=\bar{\Phi}(x, y)+\sum w_{i} \Phi_{i}(x, y)
$$

as its edge strength function. Fig. 3 illustrates the concept for the edge strength functions computed from the set of images shown in Fig. 4.

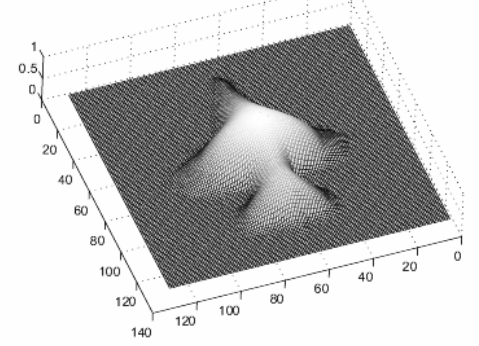

(a)

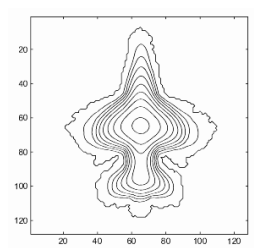

(b)

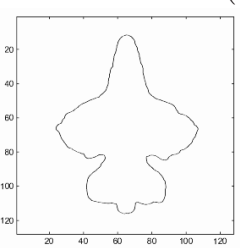

(c)

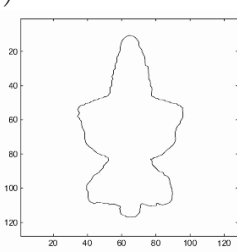

(d)

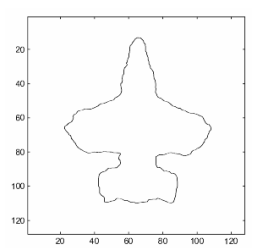

(e)

Fig. 3. (a) Mean edge strength function extracted from the set of airplane images shown in Fig. 4. (b) Corresponding level curves. (c)-(e) 1-level curves of $\Phi_{\mathbf{w}}$ for three different choices of $\mathbf{w}$. 


\section{$1+1 \pm 1$ \\ $11+1+1$}

Fig. 4. Set of airplane shapes taken from [11].

\section{Shape Energy: Similarity of the embedding surface}

In the previous section, a representation for the shape prior in terms of a mean edge strength function and principle components is developed. Now we will discuss how this representation captured by equation (4) can be used to integrate prior shape information into Ambrosio-Tortorelli functional. The simplest solution is adding two energies to arrive at a combined energy:

$$
E=E_{A T}+\mu E_{\text {shape }}
$$

where $\mu$ is the parameter which enforces the shape similarity of the embedding surface in the overall segmentation process.

A straight forward choice for shape energy is simply

$$
E_{\text {shape }}\left(v, t_{x}, t_{y}, h, \theta, \mathbf{w}\right)=\iint_{R}\left(v-T\left(\Phi_{\mathbf{w}}\right)\right)^{2} d x d y
$$

where the pose transformation function $T$ applied to the shape prior $\Phi_{\mathbf{w}}$ is defined as follows:

$$
\left[\begin{array}{c}
x^{\prime} \\
y^{\prime} \\
T\left(\Phi_{\mathbf{w}}\right)
\end{array}\right]=\underbrace{\left[\begin{array}{lll}
h & 0 & 0 \\
0 & h & 0 \\
0 & 0 & 1
\end{array}\right]}_{\text {scale }} \underbrace{\left[\begin{array}{ccc}
\cos \theta & -\sin \theta & 0 \\
\sin \theta & \cos \theta & 0 \\
0 & 0 & 1
\end{array}\right]}_{\text {rotation }}\left[\begin{array}{c}
x \\
y \\
\Phi_{\mathbf{w}}
\end{array}\right]+\underbrace{\left[\begin{array}{c}
t_{x} \\
t_{y} \\
0
\end{array}\right]}_{\text {translation }}
$$

However, we observed that such a straight forward choice may cause segmentation process to trap into local minima. Hence we considered the normalized difference:

$$
E_{\text {shape }}\left(v, t_{x}, t_{y}, h, \theta, \mathbf{w}\right)=\frac{\iint_{R}\left(v-T\left(\Phi_{\mathbf{w}}\right)\right)^{2} d x d y}{\iint_{R}\left(v+T\left(\Phi_{\mathbf{w}}\right)\right)^{2} d x d y}
$$

Upon casting the problem into a discrete setting, we arrive to the following minimization problem: 


$$
\begin{array}{r}
\min E\left(u, v, t_{x}, t_{y}, h, \theta, \mathbf{w}\right)=\sum_{i=1}^{\text {width height }} \sum_{j=1} \beta\left(u_{i, j}-g_{i, j}\right)^{2}+\alpha\left(1-v_{i, j}\right)^{2}\left(u_{x}^{2}+u_{y}^{2}\right) \\
+\frac{\rho}{2}\left(v_{x}^{2}+v_{y}^{2}\right)+\frac{v_{i, j}{ }^{2}}{2 \rho} \\
+\mu \frac{\sum_{i=1}^{\text {width }} \sum_{j=1}^{\text {height }}\left(v_{i, j}-T\left(\Phi_{\mathbf{w}}\right)_{i, j}\right)^{2}}{\sum_{i=1}^{\text {width }} \sum_{j=1}^{\text {height }}\left(v_{i, j}+T\left(\Phi_{\mathbf{w}}\right)_{i, j}\right)^{2}}(9)
\end{array}
$$

subject to constraints

$$
\begin{array}{r}
0 \leq u_{i, j} \leq 255 \\
0 \leq v_{i, j} \leq 1
\end{array}
$$

where $u_{x}, u_{y}, v_{x}, v_{y}$ are the central difference approximations for $x$ and $y$ derivatives of $u_{i, j}$ and $v_{i, j}$ respectively:

$$
\begin{aligned}
& u_{x}=\frac{u_{i+1, j}-u_{i-1, j}}{2} u_{y}=\frac{u_{i, j+1}-u_{i, j-1}}{2} \\
& v_{x}=\frac{v_{i+1, j}-v_{i-1, j}}{2} v_{y}=\frac{v_{i, j+1}-v_{i, j-1}}{2}
\end{aligned}
$$

Our algorithm recovers both pose transformation parameters $t_{x}, t_{y}, \theta, h$ and shape variability parameters $\mathbf{w}$ simultaneously along with a piecewise smooth approximation of the image $u$ and the corresponding edge strength function $v$. These parameters are evaluated via gradient descent equations obtained by minimizing the energy functional with respect to each parameter. These equations are given in the appendix.

We can summarize our overall algorithm as follows:

1. Take an input image $g$ and a set of pose and scale aligned prior edge strength functions $v_{p_{1}}, \cdots, v_{p_{n}}$.

2. Using $v_{p_{1}}, \cdots, v_{p_{n}}$, determine mean edge strength function $\bar{\Phi}$ and the principal components $\Phi_{1}, \cdots, \Phi_{n}$.

3. Initialize evolving image $u$ with $g$.

4. Initialize the edge strength function using the following equation:

$$
v_{0}=\frac{2 \alpha \rho\|\nabla u\|^{2}}{1+2 \alpha \rho\|\nabla u\|^{2}}
$$

5. Set initial values for pose transformation parameters $t_{x}, t_{y}, \theta, h$.

6. Set initial values of shape variability parameters $w_{1}, \cdots, w_{n}$.

7. Update $u$ according to the gradient descent equation (10).

8. Update $v$ according to the gradient descent equation equation (11).

9. Update $t_{x}$ and $t_{y}$ using the equations (12) and (13) respectively.

10. Update $h$ using equation (14).

11. Update $\theta$ using equation (15).

12. Compute the new shape variability parameters $w_{1}, \cdots, w_{n}$ using equation (16).

13. Repeat steps 7-12 until convergence. 


\section{Segmentation Results}

We demonstrate the segmentation results of our algorithm on various images. If the image to be segmented contains a shape that does not exhibits variations other than pose, we can use a single edge strength function of the shape as the shape prior. Otherwise, to capture the shape variability, we perform KarhunenLoeve Transform on a set of edge strength functions generated from pose and scale aligned shapes of similar type. In our experiments, we use $\mu=1$ unless otherwise stated. In order to prevent over-smoothing, we use a small smoothing radius, i.e. $\sqrt{\frac{\alpha}{\beta}}=0.1$. Typical $\rho$ values are $8,16,32$.

Consider the 'hand' shape shown in Fig. 5(a), its edge strength function computed with $\rho=8$ (given in Fig. 5(b)) is used as shape prior. Fig. 6(a) is generated from the grayscale 'hand' image by performing some translation, rotation and scaling. While the initial 1-level curve of the prior edge strength function is shown in Fig. 6(b), the final segmentation result is presented in Fig. $6(\mathrm{c})$. The recovered transformation parameters are $t_{x}=4.4492, t_{y}=-7.3222$, $\theta=24.6474^{\circ}, h=1.2513$.

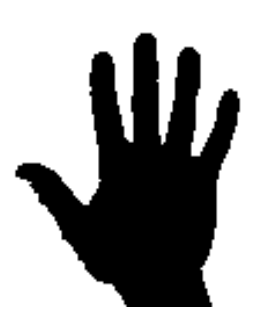

(a)

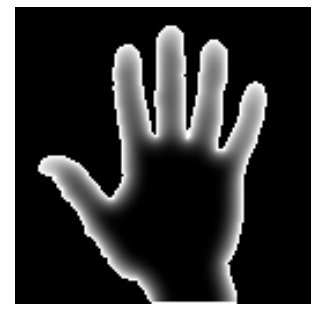

(b)

Fig. 5. (a) Prior hand shape. (b) Corresponding edge strength function computed with $\rho=8$.

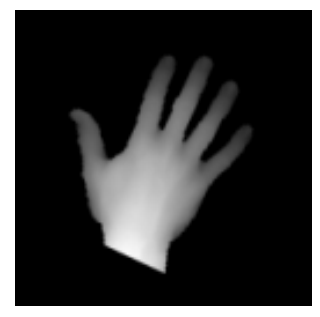

(a)

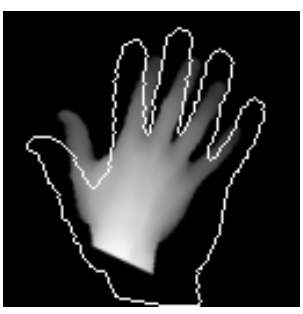

(b)

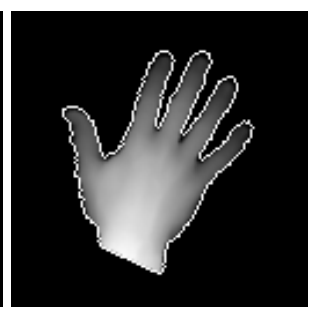

(c)

Fig. 6. Segmentation of a hand image. (a) Input image. (b) Initial 1-level curve of prior edge strength function. (c) Segmentation result. 
We next consider a case with both occlusion and missing part. Fig. 7(a) is generated from the 'hand' image shown in Fig. 6(a). The thumb is occluded by a ring type shape and some part of the pointer finger is cut off. Fig. 7(b) shows the initial 1-level curve of the prior edge strength function, Fig. 7(c) shows the final segmentation result. The recovered transformation parameters are $t_{x}=4.2945$, $t_{y}=-5.9638, \theta=25.3761^{\circ}, h=1.2810$. Instead of taking $\mu$ constant and equal to 1 , if we increase its value throughout the iterations, we can speed up the recovery process of the transformation parameters. The missing and occluded parts become apparent in the evolving edge strength function in less number of iterations(see Fig. 8).

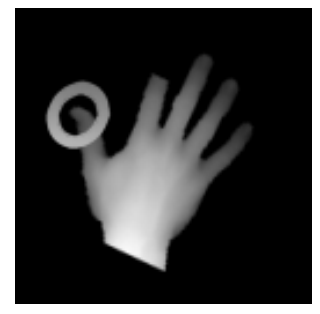

(a)

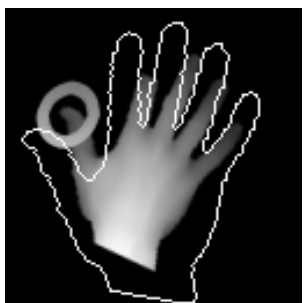

(b)

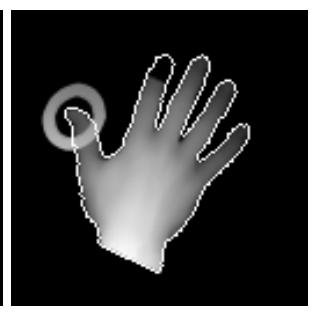

(c)

Fig. 7. Segmentation of a hand image with both occlusion and missing part. (a) Input image. (b) Initial 1-level curve of prior edge strength function. (c) Segmentation result.

Fig. 9(a) is generated from the 'hand' image shown in Fig. 6(a) by adding a noise. While Fig. 9(b) shows the initial 1-level curve of the prior edge strength function, Fig. 9(c) show the final segmentation result. The recovered transformation parameters are $t_{x}=4.3475, t_{y}=-6.4416, \theta=24.9079^{\circ}, h=1.2719$.

To demonstrate that our algorithm can handle shape variability in segmentation process, we have used the set of 'airplane' shapes shown in Fig. 4. After extracting the edge strength functions of each image in this data set, the mean edge strength function(see Fig. 3(a)) and the principal components are computed which are used to define the shape prior. For the 'airplane' images shown in Fig. 10(a) and 11(a), initial estimates of the boundary obtained from the mean edge strength function and segmentation results are shown in 10(b)-(c) and 11(b)-(c) respectively. For the first 'airplane' image the recovered parameters are $t_{x}=-3.6578, t_{y}=-3.3881, \theta=22.9083^{\circ}, h=0.9522$ and coefficients of the principle components, $\mathbf{w}=[-0.4094,-0.1463,-0.4080,-0.0919$, $0.1621,0.0832,0.1295,0.2282,0.0556,0.0264,0.0390,0.0053]$. For the second one $t_{x}=16.8324, t_{y}=-3.7704, \theta=10.7317^{\circ}, h=1.5759$ and coefficients of the principle components, $\mathbf{w}=[-0.3846,-0.0776,-0.1361,0.3473,-0.1117$, $-0.0737,-0.0053,-0.1174,0.0958,-0.1269,0.1143,-0.1388$.

Fig. 1(c)-(d) illustrates a case where the prior is given in the form of a line drawing with junctions. Since the curve $\Gamma_{p}$ is no longer a simple closed curve, 


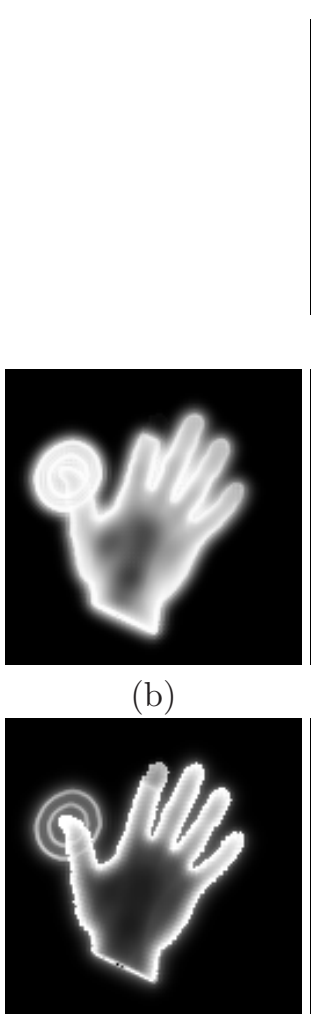

(e)

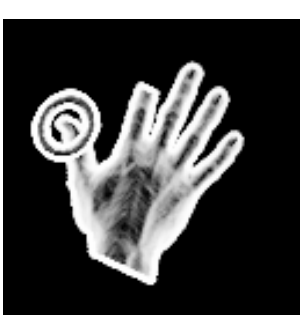

(a)

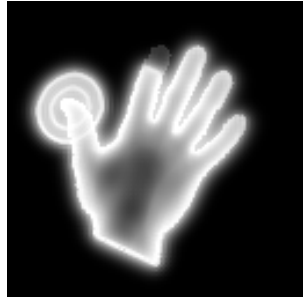

(c)

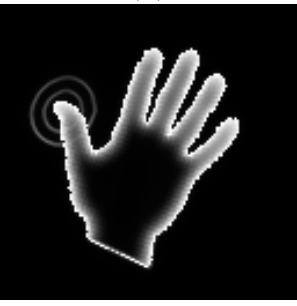

(f)

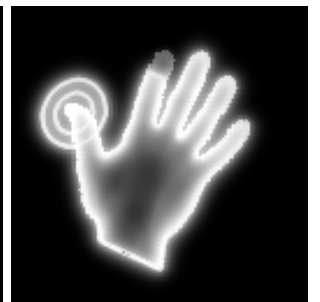

(d)

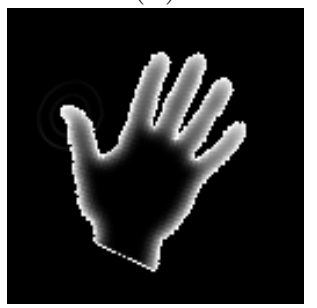

(g)

Fig. 8. Evolution of the edge strength function of the hand image given in Fig. 7 (a) throughout iterations. (a) $t=0$. (b) $t=32$. (c) $t=36$. (d) $t=40$. (e) $t=44$. (f) $t=48$. (g) $t=52$.

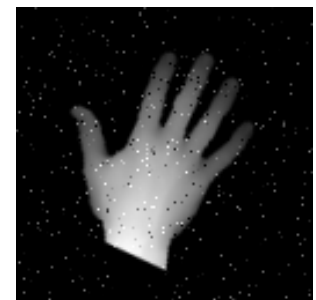

(a)

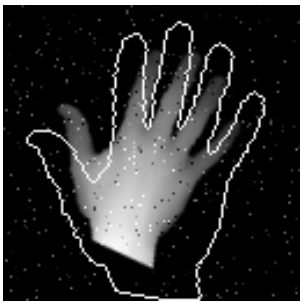

(b)

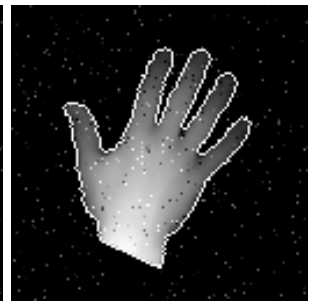

(c)

Fig. 9. Segmentation of a noisy hand image. (a) Input image. (b) Initial 1-level curve of prior edge strength function. (c) Segmentation result. 


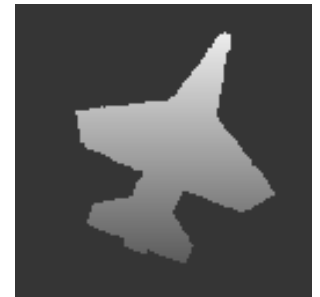

(a)

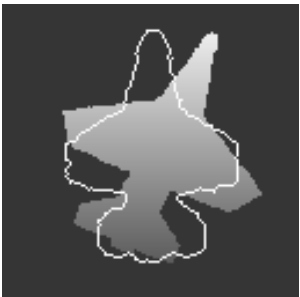

(b)

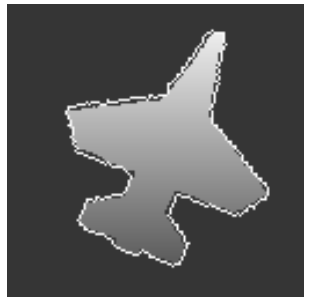

(c)

Fig. 10. Segmentation of an airplane image. (a) Input image. (b) Initial estimate of the boundary obtained from the mean edge strength function. (c) Segmentation result.

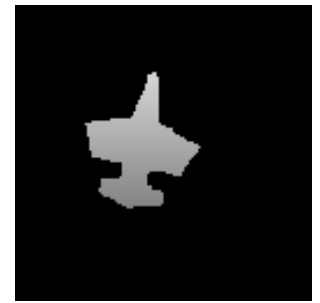

(a)

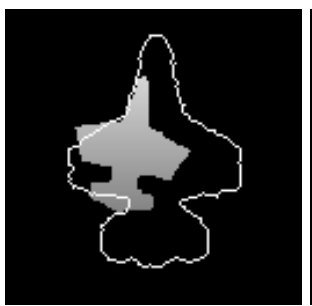

(b)

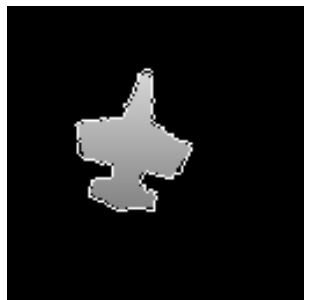

(c)

Fig. 11. Segmentation of another airplane image. (a) Input image. (b) Initial estimate of the boundary obtained from the mean edge strength function. (c) Segmentation result. 
a level set formulation can not be devised. As Fig. 12 illustrates, the algorithm is able to extract both blobs simultaneously. The recovered transformation parameters are $t_{x}=-1.5529, t_{y}=6.3731, \theta=-30.6071^{\circ}, h=1.1079$.

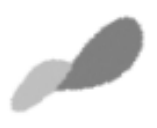

(a)

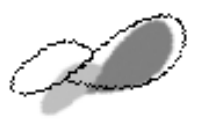

(b)

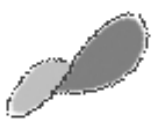

(c)

Fig. 12. (a) Input image. (b) Initial 1-level curve of prior edge strength function. (c) Segmentation result.

\section{Summary}

We have demonstrated the potential use of Ambrosio-Tortorelli edge strength function as an aid for incorporating shape priors into image segmentation. For large $\rho$ values the edge strength function $v$ provides a representation equivalent to level set representation without explicitly requiring 2 phases(inside and outside). Differential properties such as the cross derivative $\frac{d}{d s}\|\nabla v\|$ captures the local symmetry information and $1-\left|\frac{d}{d s}\|\nabla v\|\right|$ may be interpreted as the symmetry strength and may be used as a weight to force the equivalence of the evolving $v$ and transformed prior more on local symmetry points. These issues will be studied further.

Acknowledgments This work is partially funded by TUBITAK-BAYG through PhD scholarships to Erkut Erdem and Aykut Erdem. The authors would like to thank Kemal Leblebicioglu and Cagri Aslan for their helpful discussions.

\section{A Gradient Descent Equations}

The piecewise smooth image $u$, the edge strength function $v$, the translation parameters $t_{x}, t_{y}$, rotation angle $\theta$, scale factor $h$, and shape variability parameters $\mathbf{w}$ are to be recovered as the minimizers of equation (9) by applying gradient descent:

$$
\frac{\partial u_{i, j}}{\partial t}=\frac{\alpha}{2}\left[\left(1-v_{i-1, j}\right)^{2}\left(u_{i, j}-u_{i-2, j}\right)-\left(1-v_{i+1, j}\right)^{2}\left(u_{i+2, j}-u_{i, j}\right)\right.
$$




$$
\begin{aligned}
& \left.+\left(1-v_{i, j-1}\right)^{2}\left(u_{i, j}-u_{i, j-2}\right)-\left(1-v_{i, j+1}\right)^{2}\left(u_{i, j+2}-u_{i, j}\right)\right] \\
& +2 \beta\left(u_{i, j}-g_{i, j}\right)
\end{aligned}
$$

$$
\begin{aligned}
\frac{\partial v_{i, j}}{\partial t}= & -2 \alpha\left(1-v_{i, j}\right)\left(u_{x}{ }^{2}+u_{y}{ }^{2}\right)+\frac{v_{i, j}}{\rho} \\
+ & \frac{\rho}{4}\left[\left(v_{i, j}-v_{i-2, j}\right)-\left(v_{i+2, j}-v_{i, j}\right)+\left(v_{i, j}-v_{i, j-2}\right)-\left(v_{i, j+2}-v_{i, j}\right)\right] \\
+ & 2 \mu\left[\frac{\left(v_{i, j}-T\left(\Phi_{\mathbf{w}}\right)_{i, j}\right)}{S}-\frac{D\left(v_{i, j}+T\left(\Phi_{\mathbf{w}}\right)_{i, j}\right)}{S^{2}}\right] \\
\frac{\partial t_{x}}{\partial t}=2 \mu & {\left[\frac{\sum_{i=1}^{\text {width }} \sum_{j=1}^{h e i g h t}\left(v_{i, j}-T\left(\Phi_{\mathbf{w}}\right)_{i, j}\right)\left(-\frac{\partial T\left(\Phi_{\mathbf{w}}\right)}{\partial x^{\prime}}\right)_{i, j}}{S}\right.} \\
& \left.-\frac{D \sum_{i=1}^{\text {width }} \sum_{j=1}^{h e i g h t}\left(v_{i, j}+T\left(\Phi_{\mathbf{w}}\right)_{i, j}\right)\left(\frac{\partial T\left(\Phi_{\mathbf{w}}\right)}{\partial x^{\prime}}\right)_{i, j}}{S^{2}}\right] \\
\frac{\partial t_{y}}{\partial t}=2 \mu & {\left[\frac{\sum_{i=1}^{\text {width }} \sum_{j=1}^{h e i g h t}\left(v_{i, j}-T\left(\Phi_{\mathbf{w}}\right)_{i, j}\right)\left(-\frac{\partial T\left(\Phi_{\mathbf{w}}\right)}{\partial y^{\prime}}\right)_{i, j}}{S}\right.} \\
& \left.-\frac{D \sum_{i=1}^{\text {width }} \sum_{j=1}^{h e i g h t}\left(v_{i, j}+T\left(\Phi_{\mathbf{w}}\right)_{i, j}\right)\left(\frac{\partial T\left(\Phi_{\mathbf{w}}\right)}{\partial y^{\prime}}\right)_{i, j}}{S^{2}}\right]
\end{aligned}
$$

$$
\begin{aligned}
\frac{\partial h}{\partial t}=2 \mu & {\left[\frac{\sum_{i=1}^{w i d t h} \sum_{j=1}^{h e i g h t}\left(v_{i, j}-T\left(\Phi_{\mathbf{w}}\right)_{i, j}\right)\left(-\frac{\partial T\left(\Phi_{\mathbf{w}}\right)}{\partial x^{\prime}} \frac{\partial x^{\prime}}{\partial h}-\frac{\partial T\left(\Phi_{\mathbf{w}}\right)}{\partial y^{\prime}} \frac{\partial y^{\prime}}{\partial h}\right)_{i, j}}{S}\right.} \\
& \left.-\frac{D \sum_{i=1}^{w i d t h} \sum_{j=1}^{h e i g h t}\left(v_{i, j}+T\left(\Phi_{\mathbf{w}}\right)_{i, j}\right)\left(\frac{\partial T\left(\Phi_{\mathbf{w}}\right)}{\partial x^{\prime}} \frac{\partial x^{\prime}}{\partial h}+\frac{\partial T\left(\Phi_{\mathbf{w}}\right)}{\partial y^{\prime}} \frac{\partial y^{\prime}}{\partial h}\right)_{i, j}}{S^{2}}\right]
\end{aligned}
$$

$$
\begin{aligned}
\frac{\partial \theta}{\partial t}=2 \mu & {\left[\frac{\sum_{i=1}^{\text {width }} \sum_{j=1}^{\text {height }}\left(v_{i, j}-T\left(\Phi_{\mathbf{w}}\right)_{i, j}\right)\left(-\frac{\partial T\left(\Phi_{\mathbf{w}}\right)}{\partial x^{\prime}} \frac{\partial x^{\prime}}{\partial \theta}-\frac{\partial T\left(\Phi_{\mathbf{w}}\right)}{\partial y^{\prime}} \frac{\partial y^{\prime}}{\partial \theta}\right)_{i, j}}{S}\right.} \\
& \left.-\frac{D \sum_{i=1}^{w i d t h} \sum_{j=1}^{h e i g h t}\left(v_{i, j}+T\left(\Phi_{\mathbf{w}}\right)_{i, j}\right)\left(\frac{\partial T\left(\Phi_{\mathbf{w}}\right)}{\partial x^{\prime}} \frac{\partial x^{\prime}}{\partial \theta}+\frac{\partial T\left(\Phi_{\mathbf{W}}\right)}{\partial y^{\prime}} \frac{\partial y^{\prime}}{\partial \theta}\right)_{i, j}}{S^{2}}\right]
\end{aligned}
$$

$$
\begin{aligned}
\frac{\partial w_{l}}{\partial t}=2 \mu & {\left[\frac{\sum_{i=1}^{\text {width }} \sum_{j=1}^{\text {height }}\left(v_{i, j}-T\left(\Phi_{\mathbf{w}}\right)_{i, j}\right)\left(-T\left(\Phi_{l}\right)_{i, j}\right)}{S}\right.} \\
& \left.-\frac{D \sum_{i=1}^{\text {width }} \sum_{j=1}^{\text {height }}\left(v_{i, j}+T\left(\Phi_{\mathbf{w}}\right)_{i, j}\right)\left(T\left(\Phi_{l}\right)_{i, j}\right)}{S^{2}}\right]
\end{aligned}
$$


where

$$
\begin{aligned}
D & =\sum_{i=1}^{\text {width height }} \sum_{j=1}^{\text {width height }}\left(v_{i, j}-T\left(\Phi_{\mathbf{w}}\right)_{i, j}\right)^{2} \\
S & =\sum_{i=1}^{\text {wi }} \sum_{j=1}\left(v_{i, j}+T\left(\Phi_{\mathbf{w}}\right)_{i, j}\right)^{2}
\end{aligned}
$$

\section{References}

1. L. Ambrosio and V. Tortorelli. On the approximation of functionals depending on jumps by elliptic functionals via $\Gamma$-convergence. Commun. Pure Appl. Math., 43(8):999-1036, 1990.

2. C. Aslan. Disconnected skeletons for shape recognition. Master's thesis, Department of Computer Engineering, Middle East Technical University, May 2005.

3. T. Chan and L. Vese. Active contours without edges. IEEE Trans. Image Processing, 10(2):266-277, 2001.

4. Y. Chen, H. Tagare, S. Thiruvenkadam, F. Huang, D. Wilson, K. S. Gopinath, R. W. Briggs, and E. A. Geiser. Using prior shapes in geometric active contours in a variational framework. Int. J. Comput. Vision, 50(3):315-328, 2002.

5. D. Cremers, F. Tischhäuser, J. Weickert, and C. Schnörr. Diffusion snakes: Introducing statistical shape knowledge into the mumford-shah functional. Int. J. Comput. Vision, 50(3):295-313, 2002.

6. M. E. Leventon, W. Eric L. Grimson, and O. D. Faugeras. Statistical shape influence in geodesic active contours. In CVPR, pages 1316-1323, 2000.

7. D. Mumford and J. Shah. Optimal approximations by piecewise smooth functions and associated variational problems. Commun. Pure Appl. Math., 42(5):577-685, 1989.

8. T. Riklin-Raviv, N. Kiryati, and N. A. Sochen. Unlevel-sets: Geometry and priorbased segmentation. In ECCV (4), pages 50-61, 2004.

9. M. Rousson and N. Paragios. Shape priors for level set representations. In ECCV (2), pages 78-92, 2002.

10. S. Tari, J. Shah, and H. Pien. Extraction of shape skeletons from grayscale images. CVIU, 66(2):133-146, May 1997.

11. A. Tsai, A. J. Yezzi, W. M. Wells III, C. Tempany, D. Tucker, A. Fan, W. E. L. Grimson, and A. S. Willsky. A shape-based approach to the segmentation of medical imagery using level sets. IEEE Trans. Med. Imaging, 22(2):137-154, 2003. 